PROCEEDINGS OF THE

AMERICAN MATHEMATICAL SOCIETY

Volume 130, Number 9, Pages 2803-2808

S 0002-9939(02)06420-1

Article electronically published on February 4, 2002

\title{
TOROIDAL SURGERIES ON HYPERBOLIC KNOTS
}

\author{
MASAKAZU TERAGAITO
}

(Communicated by Ronald A. Fintushel)

\begin{abstract}
For a hyperbolic knot $K$ in $S^{3}$, a toroidal surgery is Dehn surgery which yields a 3 -manifold containing an incompressible torus. It is known that a toroidal surgery on $K$ is an integer or a half-integer. In this paper, we prove that all integers occur among the toroidal slopes of hyperbolic knots with bridge index at most three and tunnel number one.
\end{abstract}

\section{INTRODUCTION}

Let $K$ be a knot in the 3 -sphere $S^{3}$, and let $E(K)=S^{3}-\operatorname{Int} N(K)$ be its exterior. A slope on $\partial E(K)$ is the isotopy class of an essential unoriented simple loop. As usual [11], the set of slopes on $\partial E(K)$ is parameterized by $\mathbb{Q} \cup\{\infty\}$ so that $1 / 0$ is the meridian slope and $0 / 1$ is the longitude slope. For a slope $r$ on $\partial E(K), K(r)$ denotes the closed orientable 3-manifold obtained by $r$-Dehn surgery on $K$. Thus $K(r)=E(K) \cup V$, where $V$ is a solid torus glued to $E(K)$ along their boundaries in such a way that $r$ bounds a disk in $V$.

Now suppose that $K$ is a hyperbolic knot, i.e. the interior of $E(K)$ has a complete hyperbolic structure. If $K(r)$ is not hyperbolic, the surgery and the slope $r$ are said to be exceptional. By the hyperbolic Dehn surgery theorem [12, $K$ has only finitely many exceptional surgeries. A closed 3-manifold is toroidal if it contains an incompressible torus. If $K(r)$ is toroidal, the surgery is said to be toroidal. Clearly, a toroidal surgery is exceptional.

There are some results on toroidal surgeries on hyperbolic knots. Gordon and Luecke 8 showed that if $K(m / n)$ is toroidal, then $|n| \leq 2$. Hence a toroidal slope on a hyperbolic knot is either an integer or a half-integer. For hyperbolic alternating knots, toroidal slopes are integers divisible by four [1] (see also [10]). In this paper, we show that all integers can occur among the toroidal slopes of hyperbolic knots.

Theorem 1.1. For any integer $r$, there exists a hyperbolic knot $K$ in $S^{3}$ such that $K(r)$ is toroidal. Furthermore, $K$ has bridge index at most three and tunnel number one.

The author would like to thank Takeshi Kaneto, Kimihiko Motegi, Hiroshi Goda and Makoto Ozawa for helpful conversations.

Received by the editors December 6, 2000 and, in revised form, April 18, 2001.

2000 Mathematics Subject Classification. Primary 57M50.

(C)2002 American Mathematical Society 


\section{Doubly SEIFERT-FIBERED KNOTS}

In this section, we will describe a construction of knots in $S^{3}$ that have toroidal surgeries done by Dean [4].

Let $H$ be a standardly embedded handlebody of genus two in $S^{3}$. Then $H^{\prime}=$ $S^{3}-\operatorname{Int} H$ is also a handlebody of genus two. Let $F=\partial H=\partial H^{\prime}$. If a knot $K$ is embedded in $F$, then $\partial N(K) \cap F$ defines a slope on $\partial E(K)$, which is called the surface slope of $K$ with respect to $F$. Note that a surface slope is always integral.

Lemma 2.1. Let $K$ be a knot in $F$, and let $r$ be the surface slope of $K$ with respect to $F$. Assume that $K$ is non-separating in $F$. Then $K(r) \cong W \cup W^{\prime}$, where $W$ $\left(W^{\prime}\right)$ is obtained from $H$ ( $H^{\prime}$ resp.) by attaching a 2-handle along $K$, and they are glued along their boundaries $\partial W$ and $\partial W^{\prime}$, which are tori.

Proof. This is a special case of [4, Lemma 2.1.1]. Let $c_{1}$ and $c_{2}$ be the curves $F \cap \partial N(K)$. Then $c_{i}$ bounds a meridian disk $D_{i}$ of the attached solid torus $V$ in $K(r)$ for $i=1,2$. Let $\widehat{F}=(F-N(K)) \cup D_{1} \cup D_{2}$. Since $K$ is non-separating in $F, \widehat{F}$ is a torus. We split $K(r)$ along $\widehat{F}$ into $W$ and $W^{\prime}$. Then $W$ and $W^{\prime}$ are homeomorphic to the described ones.

For non-zero integers $m$ and $n$, let $G_{m, n}$ denote the group $\left\langle x, y \mid x^{m} y^{n}=1\right\rangle$. An element in a free group is primitive if it is a part of a basis. An element $w$ in the free group $\langle x, y\rangle$ is $(m, n)$ Seifert-fibered if $\langle x, y \mid w=1\rangle \cong G_{m, n}$. If $|m|=1$ or $|n|=1$, then $G_{m, n} \cong \mathbb{Z}$.

If a knot $K$ in $F$ represents a Seifert-fibered element of $\pi_{1}(H)$, then we say that $K$ is Seifert-fibered with respect to $H$. In particular, if $K$ represents a primitive element of $\pi_{1}(H)$, then $K$ is said to be primitive with respect to $H$. Also, if $K$ is Seifert-fibered with respect to both of $H$ and $H^{\prime}$, then it is said to be doubly Seifertfibered with respect to $F$. Note that the abelianization of $G_{m, n}$ is $\mathbb{Z} \oplus \mathbb{Z}(m, n)$, where $(m, n)$ is the greatest common divisor of $m$ and $n$. Therefore if $K$ is Seifert-fibered with respect to $H$, say, then $K$ is non-separating on $F=\partial H$.

Lemma 2.2. If a knot $K$ on $\partial H$ is $(m, n)$ Seifert-fibered with respect to $H$ for $|m|,|n| \geq 2$, then the manifold $W$ obtained by adding a 2-handle to $H$ along $K$ is a Seifert fibered manifold with incompressible boundary.

Proof. Note that $W$ has Heegaard genus two. By additivity of Heegaard genus (see [3]), $W$ is irreducible, since $\pi_{1}(W)=G_{m, n}$. Hence $W$ is Haken. Then $W$ is a Seifert fibered manifold by [13], since $G_{m, n}$ has a nontrivial center. The last part follows from the fact that the only Seifert fibered manifold with non-empty compressible boundary is a solid torus. See also [4, Lemma 2.2.1].

Lemma 2.3. Let $K$ be a doubly Seifert-fibered knot in $F$ with surface slope $r$. Then $K(r)$ is toroidal.

Proof. This immediately follows from Lemmas 2.1] and 2.2.

\section{Proof of Theorem 1.1}

Let $r$ be an integer. If $r$ is a toroidal surgery on a knot $K$, then $-r$ is one on the mirror image of $K$. Therefore we may assume that $r \geq 0$. 


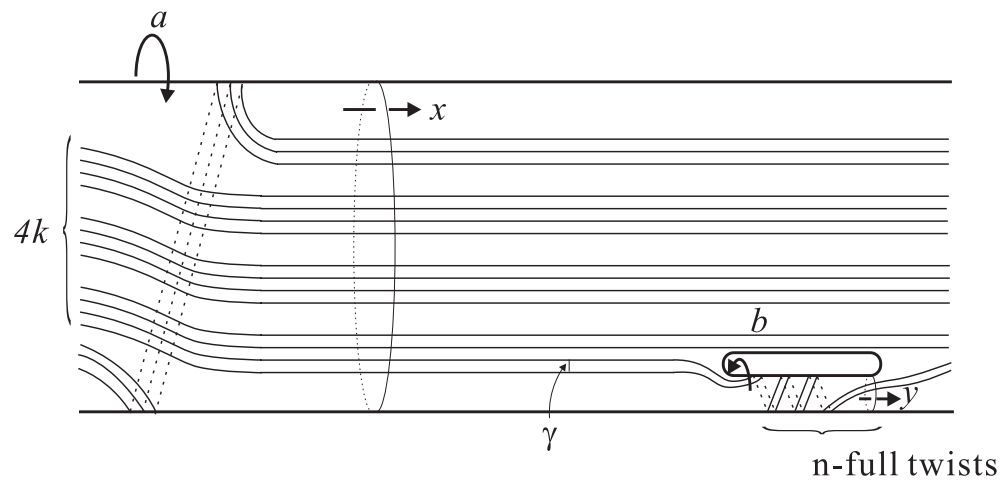

Figure 1. $K(4 k+3,3,2, n)$

We will divide the proof into three cases.

Case 1. $r \equiv 0(\bmod 4)$.

Let $K\left(b_{1}, b_{2}\right)$ be the 2-bridge knot corresponding to a continued fraction $\left[b_{1}, b_{2}\right]$. Then $K(2,-2)$ is the figure-eight knot, and 0 and 4 are toroidal surgeries [12. If $r \geq 8, K(3, r / 2)$ is hyperbolic, and $r$ gives a toroidal surgery [2, Theorem 1.1(2)]. Note that any 2-bridge knot has tunnel number one.

Case 2. $r \equiv \pm 1(\bmod 4)$.

Let $k \geq 1, k \not \equiv 0(\bmod 3)$ and $n \leq-2$. Let $K(4 k+3,3,2, n)$ be the twisted torus knot lying on $H$. It is obtained from the torus knot of type $(4 k+3,3)$ by adding $n$-full twists on two strands that are parallel in the standard torus knot picture 4. See Figure1, where the two ends are glued to form $H$. We see that the surface slope with respect to $\partial H$ is $3(4 k+3)+4 n$. As a knot in $S^{3}$, it is isotopic to $K(3,4 k+3,2, n)$, and hence it has bridge index at most three. Also, it is clear that the arc $\gamma$ shown in Figure 1 is an unknotting tunnel.

Let $\{x, y\}$ and $\{a, b\}$ be the bases of $\pi_{1}(H)$ and $\pi_{1}\left(H^{\prime}\right)$, respectively, as in Figure 1 The following two lemmas are checked straightforwardly.

Lemma 3.1. In $\pi_{1}(H)$,

$$
K(4 k+3,3,2, n) \text { represents }\left\{\begin{array}{llll}
x^{\frac{8 k+7}{3}} y x^{\frac{4 k+2}{3}} y & \text { if } & k \equiv 1 \quad(\bmod 3), \\
x^{\frac{8 k+5}{3}} y x^{\frac{4 k+4}{3}} y & \text { if } & k \equiv 2 \quad(\bmod 3) .
\end{array}\right.
$$

Lemma 3.2. In $\pi_{1}\left(H^{\prime}\right), K(4 k+3,3,2, n)$ represents $a^{2} b^{-n} a b^{-n}$.

Lemma 3.3. With respect to $H$,

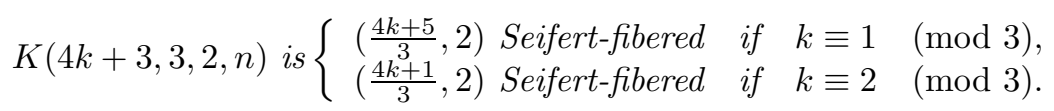

Proof. We prove the case where $k \equiv 1(\bmod 3)$. The other case is similar.

$$
\begin{aligned}
\left\langle x, y \mid x^{\frac{8 k+7}{3}} y x^{\frac{4 k+2}{3}} y=1\right\rangle & =\left\langle x, y \mid x^{\frac{4 k+5}{3}}\left(x^{\frac{4 k+2}{3}} y\right)^{2}=1\right\rangle \\
& =\left\langle x, y, z \mid x^{\frac{4 k+5}{3}} z^{2}=1, z=x^{\frac{4 k+2}{3}} y\right\rangle \\
& =\left\langle x, z \mid x^{\frac{4 k+5}{3}} z^{2}=1\right\rangle .
\end{aligned}
$$




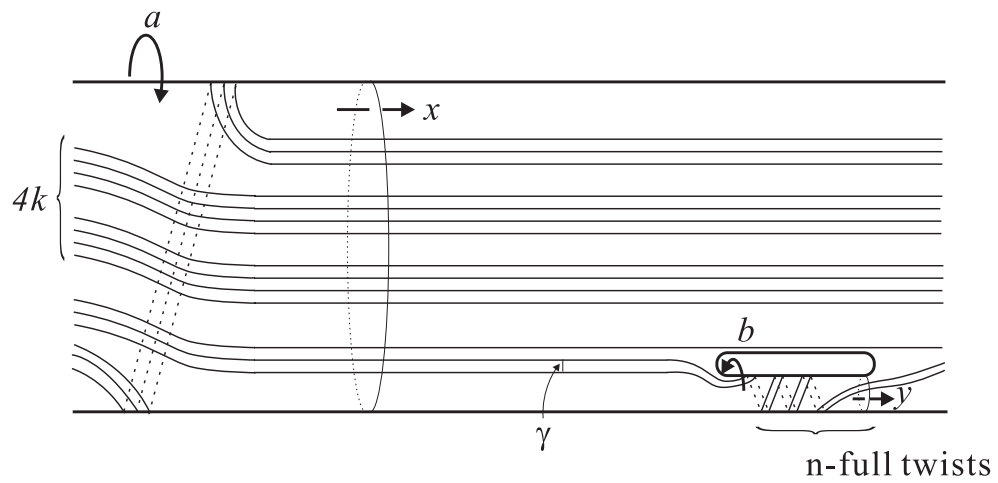

Figure $2 . K(4 k+6,3,2, n)$

Lemma 3.4. $K(4 k+3,3,2, n)$ is $(3, n)$ Seifert-fibered with respect to $H^{\prime}$.

Proof.

$$
\begin{aligned}
\left\langle a, b \mid a^{2} b^{-n} a b^{-n}=1\right\rangle & =\left\langle a, b \mid a\left(a b^{-n}\right)^{2}=1\right\rangle \\
& =\left\langle a, b, c \mid a c^{2}=1, c=a b^{-n}\right\rangle \\
& =\left\langle b, c \mid c b^{n} c^{2}=1\right\rangle \\
& =\left\langle b, c \mid c^{3} b^{n}=1\right\rangle .
\end{aligned}
$$

Proposition 3.5. For $K(4 k+3,3,2, n)$, the surface slope $3(4 k+3)+4 n$ with respect to $F$ is toroidal. Therefore the knot is hyperbolic.

Proof. The knot $K(4 k+3,3,2, n)$ is doubly Seifert-fibered by Lemmas 3.3 and 3.4 Hence the surface slope is toroidal by Lemma 2.3. Since it has bridge index at most three, it is either a torus knot or a hyperbolic knot. But a torus knot has no non-zero toroidal surgery. (In fact, 0-surgery on the trefoil is the only toroidal surgery for torus knots. See [9].) Thus the knot is hyperbolic.

For a given integer $r>0$ such that $r \equiv 1(\bmod 4)$, we choose $k$ so that $r \leq$ $12 k+1, k \neq \equiv 0(\bmod 3)$. Then the knot $K(4 k+3,3,2, n), n=-2-\frac{(12 k+1)-r}{4}$, has the surface slope $r$ exactly. If $r \equiv-1(\bmod 4)$, then consider the knot $K(7,3,2, n)$ with $n=-6+\frac{3-r}{4}$. Then it has the surface slope $-r$. Hence its mirror image is a desired one.

As stated before, if a hyperbolic 2-bridge knot has a toroidal slope, then the slope is an integer divisible by 4 [1. Therefore our knots are 3-bridge.

Case 3. $r \equiv 2(\bmod 4)$.

Let $k \geq 1, k \not \equiv 0(\bmod 3)$ and $n \leq-2$. Let $K(4 k+6,3,2, n)$ be the twisted torus knot lying on $H$. See Figure 2 We see that the surface slope with respect to $\partial H$ is $3(4 k+6)+4 n$.

As in Case 2, the knot has bridge index at most three, and tunnel number one. We use the same bases of $\pi_{1}(H)$ and $\pi_{1}\left(H^{\prime}\right)$ as in Case 1 .

The following two lemmas are checked straightforwardly. 
Lemma 3.6. In $\pi_{1}(H)$,

$$
K(4 k+6,3,2, n) \text { represents }\left\{\begin{array}{lll}
x^{\frac{8 k+13}{3}} y x^{\frac{4 k+5}{3}} y & \text { if } k \equiv 1 \quad(\bmod 3), \\
x^{\frac{8 k+11}{3}} y x^{\frac{4 k+7}{3}} y & \text { if } k \equiv 2 \quad(\bmod 3) .
\end{array}\right.
$$

Lemma 3.7. In $\pi_{1}\left(H^{\prime}\right), K(4 k+6,3,2, n)$ represents $a^{2} b^{-n} a b^{-n}$.

The next lemmas are proved by the same way as in the proofs of Lemmas 3.3 and 3.4 .

Lemma 3.8. With respect to $H$,

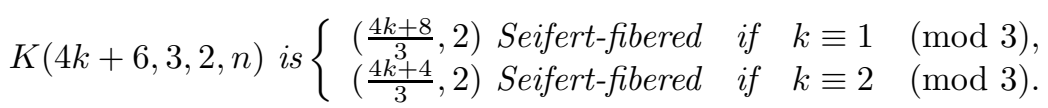

Lemma 3.9. $K(4 k+6,3,2, n)$ is $(3, n)$ Seifert-fibered with respect to $H^{\prime}$.

Proposition 3.10. For $K(4 k+6,3,2, n)$, the surface slope $3(4 k+6)+4 n$ with respect to $F$ is toroidal. Therefore the knot is hyperbolic.

Proof. The arguments in the proof of Proposition 3.5 work well.

For a given integer $r>0$ such that $r \equiv 2(\bmod 4)$, we choose $k$ so that $r \leq$ $12 k+10, k \not \equiv 0(\bmod 3)$. Then the knot $K(4 k+6,3,2, n), n=-2-\frac{(12 k+10)-r}{4}$, has the surface slope $r$. As in Case 2, the knot is 3-bridge.

Thus we have proved Theorem 1.1

Remark 3.11. Eudave-Muñoz [5] gave infinitely many hyperbolic knots with halfinteger toroidal surgeries. For example, the $(-2,3,7)$-pretzel knot has a toroidal slope $37 / 2$. Among his knots, $k(l, m, n, 0)$ (in his notation) has a non-integral toroidal slope

$$
-\frac{1}{2}-l+l^{2} m+2 l m-2 l^{2} m^{2}+(2 l m-1)^{2} n
$$

Indeed, $k(3,1,1,0)$ is the $(-2,3,7)$-pretzel knot. Also, $k(l, m, 0, p)$ has a nonintegral toroidal slope

$$
-\frac{1}{2}-l+l^{2} m+2 l m-2 l^{2} m^{2}+(2 l m-1-l)^{2} p .
$$

See also 6] Propositions 5.3, 5.4]. Since his knots are expected to give all hyperbolic knots with non-integral toroidal surgeries [7, it seems to be reasonable to conjecture that not all $n / 2$ can be realized as toroidal slopes of hyperbolic knots. In fact, we may conjecture that $|n / 2| \geq 37 / 2$.

\section{REFERENCES}

1. S. Boyer and X. Zhang, Cyclic surgery and boundary slopes, in Geometric topology (Athens, GA, 1993), 62-79, AMS/IP Stud. Adv. Math., 2.1, Amer. Math. Soc., Providence, RI, 1997. MR 98i:57005

2. M. Brittenham and Y.Q. Wu. The classification of exceptional surgeries on 2-bridge knots, Comm. Anal. Geom. 9 (2001), 97-113. CMP 2001:07

3. A. Casson and C. McA. Gordon, Reducing Heegaard splittings, Topology Appl. 27 (1987), 275-283. MR 89c:57020

4. J. Dean, Hyperbolic knots with small Seifert-fibered Dehn surgeries, Ph.D. Thesis, The University of Texas at Austin, 1996.

5. M. Eudave-Muñoz, Non-hyperbolic manifolds obtained by Dehn surgery on hyperbolic knots, in Geometric topology (Athens, GA, 1993), 35-61, AMS/IP Stud. Adv. Math., 2.1, Amer. Math. Soc., Providence, RI, 1997. MR 98i:57007 
6. M. Eudave-Muñoz, On hyperbolic knots with Seifert fibered Dehn surgeries, preprint.

7. C. McA. Gordon, Dehn filling: a survey, Knot theory (Warsaw, 1995), 129-144, Banach Center Publ., 42, Polish Acad. Sci., Warsaw, 1998. MR 99e:57028

8. C. McA. Gordon and J. Luecke, Dehn surgeries on knots creating essential tori, I, Communications in Analysis and Geometry 3 (1995), 597-644. MR 96k:57003

9. L. Moser, Elementary surgery along a torus knot, Pacific J. Math. 38 (1971), 737-745. MR 52:4287

10. R. Patton, Incompressible punctured tori in the complements of alternating knots, Math. Ann. 301 (1995), 1-22. MR 95k:57011

11. D. Rolfsen, Knots and links, Mathematics Lecture Series, 7, Publish or Perish, Inc., Berkeley, Calif., 1976. MR 95c:57018

12. W. Thurston, The geometry and topology of 3-manifolds, Princeton University, 1978.

13. F. Waldhausen, Gruppen mit Zentrum und 3-dimensionale Mannigfaltigkeiten, Topology 6 (1967), 505-517. MR 38:5223

Department of Mathematics and Mathematics Education, Faculty of Education, Hiroshima University, 1-1-1 Kagamiyama, Higashi-Hiroshima 739-8524, JaPAN

E-mail address: teragai@hiroshima-u.ac.jp 